\title{
Development and validation of a model to describe the bearings interaction in rotating machines due to elastic supporting structures
}

\author{
Enrico Meli, Giovanni Pallini, Andrea Rindi, and Stefano Rossin \\ Department of Industrial Engineering \\ University of Florence \\ via di S.Marta 3, 50139 Florence, Italy \\ enrico.meli@unifi.it,giovanni.pallini@unifi.it, \\ andrea.rindi@unifi.it, stefano.rossin@ge.it \\ http://www.dief .unifi.it/ \\ http://www.ge.com/
}

\begin{abstract}
This work focuses on the effects of the flexible supporting structures and on the bearing interaction caused by their elastic deflection on the whole rotor-substructures system. More particularly, a careful theoretical and experimental analysis is performed to understand how the supporting structures influence the rotors behaviour through the actions of the machine bearings. To this end, differently from many standard approaches, this study considers a model of the whole rotating machine [1], taking into account the coupling of the dynamic behavior of the different system components. The whole FEM model has been implemented in the ANSYS [2] simulation environment. The main goal of this research, is to offer an optimal balance between efficiency and accuracy allowing the modelling of the real phisical complex system and simultaneously the reduction of calculation times. The whole analysis has been developed and validated in cooperation with General Electric S.p.A. which provided the technical and experimental data related to some tests recently performed in Massa-Carrara (Italy) on a benchmark turbocompressor machine.
\end{abstract}

Keywords: rotating machines, supporting structures, bearings, dynamical, bearing interaction

\section{Introduction}

FEM theory offers numerous solutions to represent rotor systems (beam and solid model, transfer function..) and many formulations of the problem have been proposed (based on Timoshenko's [3] and Bernoulli's [4] theory). In this paper the attention is focused on the implementation of a reduced order model for baseplates based on modal dynamic reduction techniques. The authors developed a first Complete model to evaluate the behaviour of the whole system (rotor and support structure) with no simplification and a second Reduced model based on 


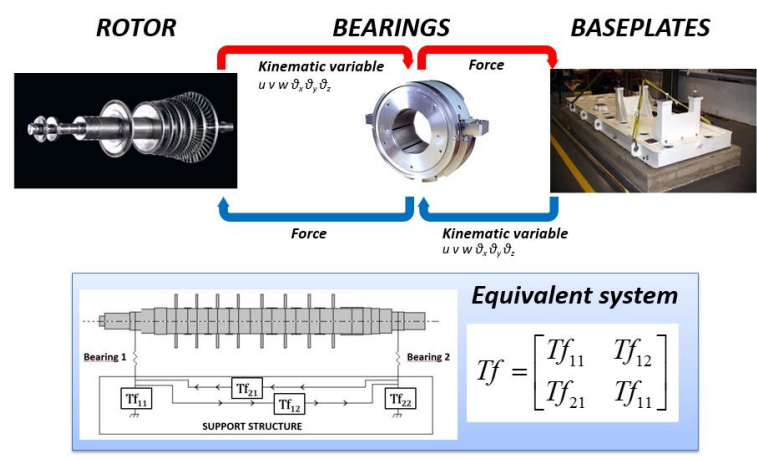

Fig. 1. General architecture step subdivisions

modal reduction, that describes in different ways the support dynamic effects. In the first Reduced model, the supporting structure has been reduced with the CMS technique without any further simplification in the dynamics of baseplate and in the bearing interaction. In the second Reduced model (three-diagonal model) the transfer function allows the coupling between bearings, employs the crosstalking $\left(T f_{12}\right.$ and $\left.T f_{21}\right)$ terms and neglect the cross-coupling terms (Fig.1) (all sub-matrices $T f_{i j}$ are diagonal). In the third Reduced model (block model), the transfer function that represents the baseplate neglects the coupling between bearings $\left(T f_{12} \equiv T f_{21} \equiv 0\right)$ but cross-coupling terms are present. In the fourth Reduced model (diagonal model) the transfer function neglects the coupling between bearings and the cross-coupling terms, so $T f_{12} \equiv T f_{21} \equiv 0$ and $T f_{11}, T f_{22}$ are diagonal matrices; this is the most reduced model. All system are capable to evaluate both steady state harmonic responses and transient analysis!!!, this paper will focus on the result of the first kind of analysis.

\section{The model: the Rotor and the Bearings}

The main component of the assembly is the rotor shaft. It is modelled with 3D beams with circular cross section and has 6 DOFs per node. The shaft is char-

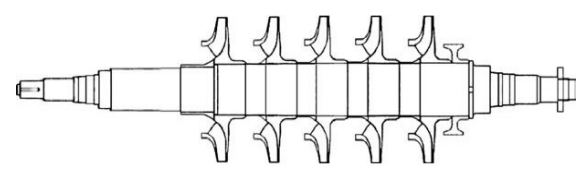

Fig. 2. Example of 5 stage centrifugal compressor

acterised by its own stiffness and mass properties. The rotor instead, that is the sum of many supplementary elements linked to the shaft (like the impellers) is characterized with lumped mass $\left(m_{x}, m_{y}, m_{z}, I_{p}, I_{t}\right)$ and consequently with the 
required mass and gyroscopic effect matrices. Finally the complete rotordynamical equation can be written as:

$$
M_{r} \ddot{\mathbf{q}}_{r}+\left(C_{r}+\omega G_{r}\right) \dot{\mathbf{q}}_{r}+K_{r} \mathbf{q}_{r}=\mathbf{F}_{r}^{b r g}+\mathbf{F}_{r}^{e x t} .
$$

In Eq.1 with the subscript $r$ it is possible to recognize all the matrices, vectors and coefficients related to the rotor, $\mathbf{F}_{r}^{b r g}$ represents the contribute of the bearings reaction forces, $\mathbf{F}_{r}^{\text {ext }}$ is the vector that gather all external loads, $M_{r}, C_{r}, K_{r}$ and $G_{r}$ respectively mass, damping, stiffness and gyroscopic effect. All degree of freedom of the system are then split into:

$$
\mathbf{q}_{r}=\left\{\mathbf{q}_{r b}^{T}, \mathbf{q}_{r g}^{T}\right\}^{T} \quad \mathbf{q}_{s}=\left\{\mathbf{q}_{s b}^{T}, \mathbf{q}_{s g}^{T}\right\}^{T},
$$

where $r$ refers to the rotor, $s$ to the support structure and the letter $b$ and $g$ respectively represent the DOFs related to the nodes belonging to the bearing interface and all the residual DOFs (general node DOF). Consequently, Eq.1 becomes:

$$
\begin{gathered}
{\left[\begin{array}{cc}
M_{r}^{b b} & M_{r}^{b g} \\
M_{r}^{g b} & M_{r}^{g g}
\end{array}\right]\left\{\begin{array}{c}
\ddot{\mathbf{q}}_{r b} \\
\ddot{\mathbf{q}}_{r g}
\end{array}\right\}+\left[\begin{array}{cc}
C_{r}^{b b} & C_{r}^{b g} \\
C_{r}^{g b} & C_{r}^{g g}
\end{array}\right]\left\{\begin{array}{c}
\dot{\mathbf{q}}_{r b} \\
\dot{\mathbf{q}}_{r g}
\end{array}\right\}+\left[\begin{array}{cc}
K_{r}^{b b} & K_{r}^{b g} \\
K_{r}^{g b} & K_{r}^{g g}
\end{array}\right]\left\{\begin{array}{c}
\mathbf{q}_{r b} \\
\mathbf{q}_{r g}
\end{array}\right\}=} \\
=\left\{\begin{array}{c}
C^{b r g}\left(\dot{\mathbf{q}}_{r b}-\dot{\mathbf{q}}_{s b}\right) \\
0
\end{array}\right\}+\left\{\begin{array}{c}
K^{b r g}\left(\mathbf{q}_{r b}-\mathbf{q}_{s b}\right) \\
0
\end{array}\right\}+\left\{\begin{array}{c}
0 \\
F_{r}^{e x t}
\end{array}\right\} .
\end{gathered}
$$

The bearing forces can be written as:

$$
\begin{aligned}
& \left\{F_{b r g}(\omega)\right\}=\left\{\begin{array}{l}
F_{x}(\omega) \\
F_{y}(\omega) \\
F_{z}(\omega)
\end{array}\right\}=\left[\begin{array}{cc}
-K_{x x}(\omega) & 0 \\
0 & -K_{y y}(\omega)-K_{y z}(\omega) \\
0 & -K_{z y}(\omega)-K_{z z}(\omega)
\end{array}\right]\left\{\begin{array}{l}
q_{x r b}-q_{x s b} \\
q_{y r b}-q_{y s b} \\
q_{z r b}-q_{z s b}
\end{array}\right\}+ \\
& +\left[\begin{array}{ccc}
-C_{x x}(\omega) & 0 & 0 \\
0 & -C_{y y}(\omega)-C_{y z}(\omega) \\
0 & -C_{z y}(\omega)-C_{z z}(\omega)
\end{array}\right]\left\{\begin{array}{l}
\dot{q}_{x r b}-\dot{q}_{x s b} \\
\dot{q}_{y r b}-\dot{q}_{y s b} \\
\dot{q}_{z r b}-\dot{q}_{z s b}
\end{array}\right\} .
\end{aligned}
$$

In Eq.4 it is possible to note that, in $K^{b r g}$ and $C^{b r g}$ matrices the action in the axial direction is uncoupled from the transversal actions. To better match the real response of the assembly $K^{b r g}(\omega)$ and $C^{b r g}(\omega)$ matrices are frequency dependent ( $\omega$ is the rotational velocity of the shaft).

\section{The model: Elastic support structure}

To model the elastic support structure It is natural to write once again the linear equations that describe the motion of the baseplate assembly (coming from a suitable FEM discretization)

$$
M_{s} \ddot{\mathbf{q}}_{s}+C_{s} \dot{\mathbf{q}}_{s}+K_{s} \mathbf{q}_{s}=\mathbf{F}_{s}^{e x t}+\mathbf{F}^{b r g} .
$$

In Eq.5 $\mathbf{F}_{s}^{e x t}$ represents a general external load, $\mathbf{F}^{b r g}$ considers the reaction forces exchanged with bearings, $M_{s}, K_{s}, C_{s}$ the mass, stiffness and damping matrices of the baseplate and six support columns. The new set of coordinate $\overline{\mathbf{q}}$ comprises 
the coordinates of the nodes related to the rotor and to the support structure $\overline{\mathbf{q}}=\left\{\mathbf{q}_{r b}^{T}, \mathbf{q}_{r g}^{T}, \mathbf{q}_{s b}^{T}, \mathbf{q}_{s g}^{T}\right\}^{T} ;$ consequently the global system equation are:

$$
\begin{gathered}
{\left[\begin{array}{cccc}
M_{r}^{b b} & M_{r}^{b g} & 0 & 0 \\
M_{r}^{g b} & M_{r}^{g g} & 0 & 0 \\
0 & 0 & M_{s}^{b b} & M_{s}^{b g} \\
0 & 0 & M_{s}^{g b} & M_{s}^{g g}
\end{array}\right]\left\{\begin{array}{c}
\ddot{\mathbf{q}}_{r b} \\
\ddot{\mathbf{q}}_{r g} \\
\ddot{\mathbf{q}}_{s b} \\
\ddot{\mathbf{q}}_{s g}
\end{array}\right\}+} \\
+\left\{\left[\begin{array}{cccc}
C_{r}^{b b} & C_{r}^{b g} & C^{b r g} & 0 \\
C_{b}^{g b} & C_{r}^{g g} & 0 & 0 \\
C^{b r g} & 0 & C_{s}^{b b} & C_{s}^{b g} \\
0 & 0 & C_{s}^{g b} & C_{s}^{g g}
\end{array}\right]+\omega\left[\begin{array}{cccc}
G_{r}^{b b} & 0 & 0 & 0 \\
0 & G_{r}^{g g} & 0 & 0 \\
0 & 0 & 0 & 0 \\
0 & 0 & 0 & 0
\end{array}\right]\right\}\left\{\begin{array}{c}
\dot{\mathbf{q}}_{r b} \\
\dot{\mathbf{q}}_{r g} \\
\dot{\mathbf{q}}_{s b} \\
\dot{\mathbf{q}}_{s g}
\end{array}\right\}+ \\
+\left[\begin{array}{cccc}
K_{r}^{b b} & K_{r}^{b g} & K^{b r g} & 0 \\
K_{r}^{g b} & K_{r}^{g g} & 0 & 0 \\
K^{b r g} & 0 & K_{s}^{b b} & K_{s}^{b g} \\
0 & 0 & K_{s}^{g b} & K_{s}^{g g}
\end{array}\right]\left\{\begin{array}{c}
\dot{\mathbf{q}}_{r b} \\
\dot{\mathbf{q}}_{r g} \\
\dot{\mathbf{q}}_{s b} \\
\dot{\mathbf{q}}_{s g}
\end{array}\right\}=\left\{\begin{array}{c}
0 \\
F_{r g} \\
0 \\
F_{s g}
\end{array}\right\}
\end{gathered}
$$

In Eq.6 $\mathbf{F}^{\text {ext }}$ comprises all the loading forces applied to the complete assembly. The Complete model equation of the assembly can then be summarised and written in the frequency domain (this time $\bar{M}, \bar{G}, \bar{K}$ and $\bar{C}$ represent the mass, the gyrospic effect, stiffness and damping matrices of the complete system):

$$
\left[-\omega^{2} \bar{M}+i \omega(\bar{C}+\omega \bar{G})+\bar{K}\right] \mathbf{U}_{0}=\mathbf{F}_{0} .
$$

\subsection{Reduced Model: Dynamic substructuring}

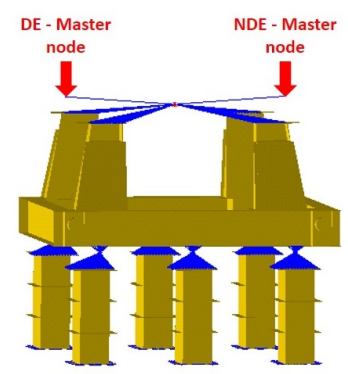

Fig. 3. Superelement graphical representation

Master DOFs have been chosen on the boundary of the support structure, as highlighted in Fig.3( nodes that represent the linking between bearings and foundations). As the goal of this analysis is to describe the flexo-torsional behaviour of the assembly, each master node comprises all the six DOFs. Compomponent Mode Synthesis (CMS [5]) is an improvement and a fusion of Static and Modal 
reduction [6] techniques that aim at obtaining a valid description of the model both in the high and the low frequency range. The biggest disadvantage of this approach is the increase of the problem size compared to the Static and Modal reduction. Furthermore CMS provides dense matrices, as modal reduction outputs and not spares. Despite all problemes, CMS guarantee excellent results with non optimal master nodes distribution as well as in case of a poor modal base. In particular, referring to the test case, master nodes have been chosen on the bearing interface located on the supporting structure $\left(q_{s b}\right)$ and slaves nodes are the residual system nodes $\left(q_{s g}\right)$.

The free interface Component Mode Synthesis allows the definition of a transformation matrix $T$ that has a larger frequency range of validity

$$
T=\left[\begin{array}{ccc}
I & 0 & 0 \\
B & \psi_{i r} & \psi_{i}
\end{array}\right],
$$

where $\psi_{i}=\psi_{s}-B \psi_{m}$ and, respectively, $\psi_{m}$ represents the matrix of the master DOFs partition; $\psi_{s}$ is the matrix of the slave DOF partition and $B$ is a part of the stiffness matrix inheritated from the static reduction; $\psi_{i r}$ is the matrix of inertia relief modes, included only if rigid body modes are present[5]. The new system coordinates becomes:

$$
\left\{\begin{array}{c}
\mathbf{q}_{s b} \\
\mathbf{q}_{s g}
\end{array}\right\}=T\left\{\begin{array}{c}
\mathbf{q}_{s b} \\
\delta
\end{array}\right\}=T \eta_{s},
$$

where $\delta$ is a truncated set of generalized modal coordinates. After the dynamic modal reduction of the supporting structure, the new equation of the Reduced system is

$$
\begin{gathered}
{\left[\begin{array}{ccc}
M_{r} & M_{r} & 0 \\
M_{r} & M_{r} & 0 \\
0 & 0 & M_{s}
\end{array}\right]\left\{\begin{array}{c}
\ddot{\mathbf{q}}_{r b} \\
\ddot{\mathbf{q}}_{r g} \\
T \ddot{\eta}_{s}
\end{array}\right\}+\left[\begin{array}{ccc}
C_{r} & C_{r} & C^{b r g} \\
C_{r} & C_{r} & 0 \\
C^{b r g} & 0 & K_{s}
\end{array}\right]\left\{\begin{array}{l}
\dot{\mathbf{q}}_{r b} \\
\dot{\mathbf{q}}_{r g} \\
T \dot{\eta}_{s}
\end{array}\right\}+} \\
+\left[\begin{array}{ccc}
K_{r} & K_{r} & K^{b r g} \\
K_{r} & K_{r} & 0 \\
K^{b r g} & 0 & K_{s}
\end{array}\right]\left[\begin{array}{c}
\mathbf{q}_{r b} \\
\mathbf{q}_{r g} \\
T \eta_{s}
\end{array}\right]=\left\{\begin{array}{c}
0 \\
\mathbf{F}_{r} \\
\mathbf{F}_{s}
\end{array}\right\}
\end{gathered}
$$

The equation of the reduced system (rotor and supporting structure) in the frequency domain is:

$$
\left(-\omega^{2} \hat{M}+i \omega\{\hat{C}+\omega \hat{G}\}+\hat{K}\right) \mathbf{U}_{0}=\mathbf{F}_{0} .
$$

This time the $\hat{M}, \hat{C}, \hat{K}, \hat{G}$ represent the matrices of the reduced model, where the contribute of the supporting structure has been reduced to the master nodes at the interface with bearings. In Tab.1 all reduced model are newly summarized

\section{Test case}

The system under investigation is part of a more complex rotor train composed of a steam turbine driver and a longer commune foundation. The steam expansion is able to generate an output of $60 \mathrm{MW}$, then an elastic coupling directly 
Table 1. Reduced model

\begin{tabular}{llcc}
\hline \multicolumn{3}{c}{ Reduced Threediagonal Block Diagonal } \\
\hline Cross coupling & $\checkmark$ & $\checkmark$ \\
Cross talking & $\checkmark$ & $\checkmark$ & \\
\hline
\end{tabular}

transmits the wrench to the centrifugal compressor. The direct elastic joint partially uncouples the transmission of lateral vibrations between the driver and the centrifugal compressor. In fact, the commune foundation is an important and critical choice for rotating machine considering the transmission of vibration along the shaft line: despite all uncoupling attempts, the elastic commune support produces the transmission of vibrations and the birth of coupling transfer function between different bearings. The bearing span is $3550 \mathrm{~mm}$, while the

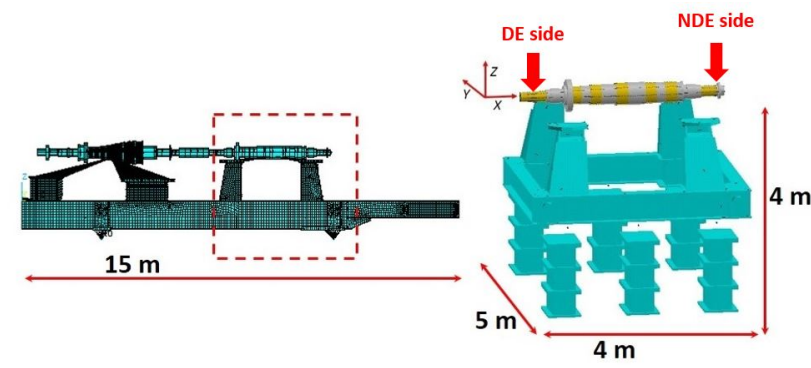

Fig. 4. Temporary baseplate

total length of the rotor is $4200 \mathrm{~mm}$ for a weight of $5530 \mathrm{Kg}$. Acronym $D E$ and $N D E$, used to mark the left and right end, come from the original system where you can recognize the location of the motion forces input ( $D E$ means Drive End, while NDE stand for Non Drive End). The centrifugal compressor operates in the range between $[4000-5775 \mathrm{rpm}]$ and, at the nominal speed, is able to get a compression ratio of 12 .

\section{Validation}

To compare the complete model and the reduced one (with full $T f$ matrix), in Tab.2 the eigenvalues of both models are reported (at the constant velocity of $5775 \mathrm{rpm}$ ). The comparison in Tab.2 confirms the high accuracy level of the reduction technique in the modal analysis. A second validation is performed evaluating the results of the model in an harmonic analysis(corresponding to a forced analysis with rotating load). The value of the unbalance rotating sinchronously with the shaft velocity is derived from the API standard

$$
U=6350 \frac{W}{N}[\mathrm{gmm}] \quad F_{\text {unbalance }}=U \omega^{2}[N]
$$


Table 2. Eigenvalue comparison - 5775 rpm

\begin{tabular}{|c|c|c|}
\hline \multicolumn{3}{|c|}{ d Complete Reduced } \\
\hline 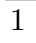 & 1001.90 & 1001.90 \\
\hline & 1079.97 & 1079.97 \\
\hline & 1908.27 & 1908.27 \\
\hline & 2191.12 & 2191.12 \\
\hline & 2221.85 & 2221.85 \\
\hline & 4575.29 & 4575.29 \\
\hline & 4840.37 & 4840.37 \\
\hline
\end{tabular}

\begin{tabular}{|c|c|c|}
\hline \multicolumn{3}{|c|}{ d Complete Reduce } \\
\hline & 5187.75 & 5187.75 \\
\hline & 591 & \\
\hline & 734 & \\
\hline 1 & 7695 & 769 \\
\hline 12 & 8247 & \\
\hline & 8609.06 & 8609.0 \\
\hline
\end{tabular}

where $\mathrm{N}$ is the maximum continuous speed (in revolution per minutes) and $W$ is the journal static weight load (in kilograms) characteristic of the deflection case. Finally, in Fig.5 it is possible to find the results of the complete and the reduced model reported in the same graphic with the experimental data of the vertical displacements measured at the drive end bearing.

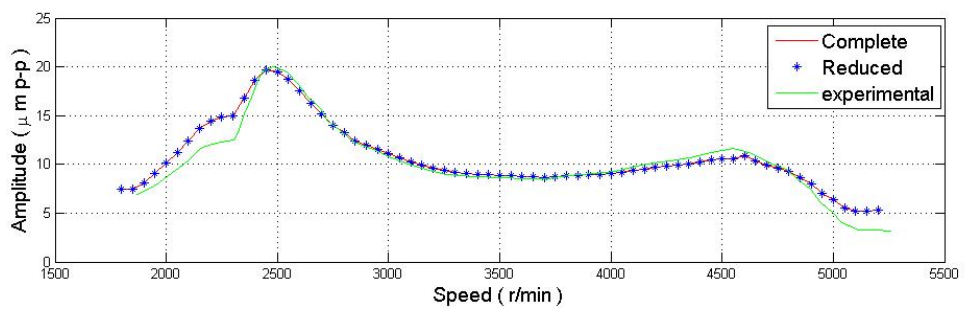

Fig. 5. Harmonic response, vertical displacements, DE bearing: comparison between reduced (full $T f$ ) and non reduced model

\section{$6 \quad$ Reduced model results}

After the validation of the reduced model method the main goal of this study is to show how the growing level of approximation in modelling the supporting structures can affect the accuracy in modelling the entire system (rotor and supporting structure). Tab.3 contains the results of the whole analysis based on the dynamic modal reduction of the supporting structure: in the first column with the Reduced model and proceding to the right where the simplification grows till the complete uncoupling of bearings dynamic with the Diagonal model. Results reported in Tab.3 confirm the birth of not existing eigenvalues along with the growth of the simplification, highlighting the minimum information needed to build an accurate transfer matrix of the baseplate. In particular the threediagonal model seems to be the simplified acceptable baseplate model. It is clear that 
Table 3. Eigenvalue comparison - 5775rpm

\begin{tabular}{lcccc}
\hline id & Reduced & Threediagonal & Block & Diagonal \\
\hline 1 & 1001.90 & 1001.91 & 1014.62 & 1014.63 \\
2 & & & & 1027.84 \\
3 & & & & 1027.85 \\
4 & 1079.97 & 1079.97 & 1097.84 & 1097.84 \\
5 & & & 1112.33 & 1112.33 \\
6 & & & & 1117.07 \\
7 & 1908.27 & 1908.27 & 1908.28 & 1908.28 \\
8 & & & 1908.29 & 1908.29 \\
9 & 2191.12 & 2191.12 & 2156.18 & 2156.18 \\
10 & 2221.85 & 2221.85 & 2230.61 & 2230.61 \\
11 & & & 2945.99 & 2946.00 \\
12 & & & 4530.56 & 4529.25 \\
13 & & & & 4556.90 \\
14 & & & & 4556.96 \\
15 & & & & 4558.35 \\
\hline
\end{tabular}

\begin{tabular}{lllll}
\hline id & Reduced & Threediagonal & Block & Diagonal \\
\hline 16 & & & 4566.32 & 4566.38 \\
17 & 4575.29 & 4575.41 & & \\
18 & & & 4834.75 & 4833.62 \\
19 & & & & 4837.90 \\
20 & & & & 4837.96 \\
21 & 4840.37 & 4840.48 & 4839.15 & 4839.01 \\
22 & & & & 4839.20 \\
23 & 5187.75 & 5187.75 & & \\
24 & & & 5214.29 & 5214.29 \\
25 & 5915.58 & 5915.58 & & \\
26 & & & 5957.30 & 5957.31 \\
27 & 7346.16 & 7346.16 & 7346.70 & 7346.70 \\
28 & & & 7347.84 & 7347.18 \\
29 & & & & 7347.84 \\
30 & 7695.72 & 7695.72 & 7695.84 & 7695.84 \\
\hline
\end{tabular}

with the accordance of eigenvalue, it is necessary to perform a similar analysis on the eigenvector of the problem. Here in particular there are some modal shapes that remain completely the same (Fig.6), independently from the level of approximation. On the other hand considering Fig. 7 there are some frequencies

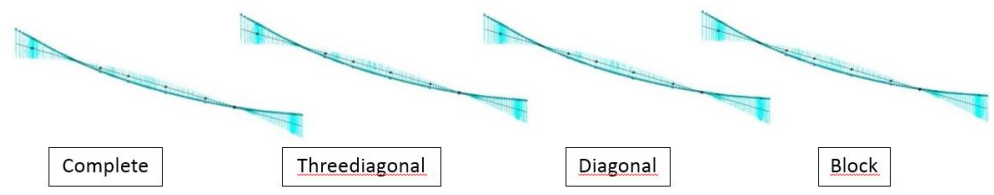

Fig. 6. Deformed shapes: 4562rpm - 76,2Hz

that all the four model are able to reproduce, but in this case, the simpler model produces a wrong deformed shape. Finally, the last error case is present

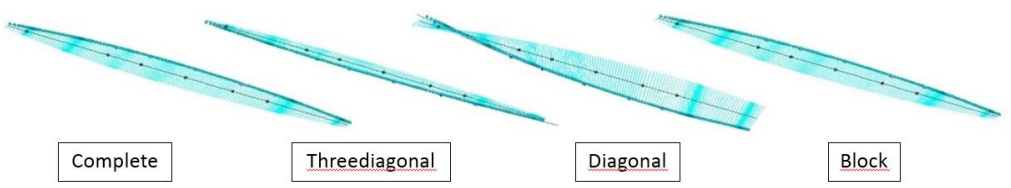

Fig. 7. Deformed shapes: 1908rpm - 31,8Hz

when the simplification of the model, obtained with modal reduction, leads to purely inconsistent frequencies and modal shapes: the block and diagonal model produces eigenvalues and eigenvector that the more accurate models (reduced and threediagonal) don't exhibit. The results of the modal shape analysis are summarized in Tab.4. Analysing the result of the unbalance response where all the reduced model are considered (reduced, threediagonal, block, diagonal) 


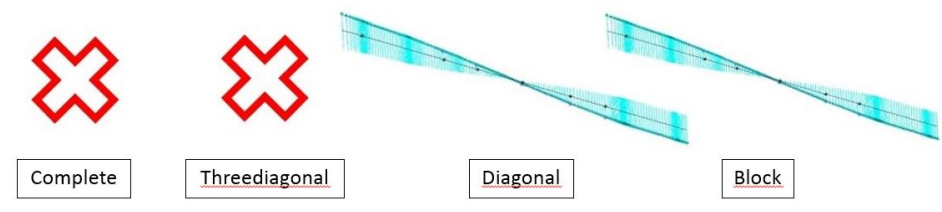

Fig. 8. Deformed shapes: 4530rpm - 75,5Hz

Table 4. Summary table

\begin{tabular}{lccc}
\hline & Threediagonal & \multicolumn{3}{c}{ Block Diagonal } \\
\hline unvaried eigenvalue & $\checkmark$ & $\checkmark$ & $\checkmark$ \\
varied deformed shapes & & $\checkmark$ & $\checkmark$ \\
not existing & $\checkmark$ & $\checkmark$ \\
\hline
\end{tabular}

(Fig.6), it is possible to see a good agreement for the first mode (a pure mode of the rotor), but the second mode (coming from the baseplate) highlights the limit of the simplificated model. Observing the second peak (Fig.6), it is possible to

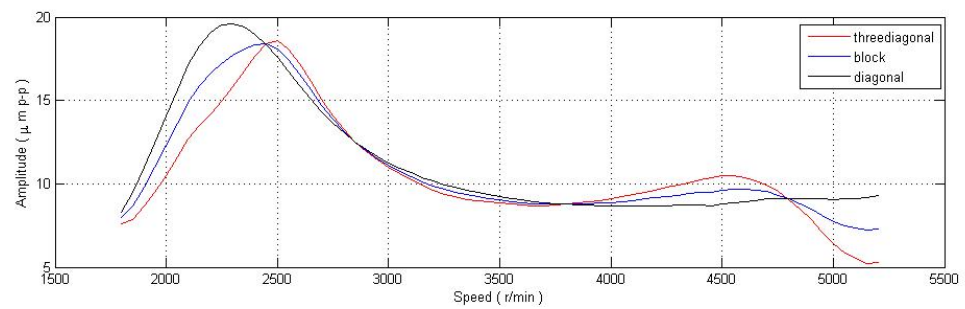

Fig. 9. Harmonic response, vertical relative displacements, DE bearing: comparison threediagonal, block and diagonal model

understand that the simpler steps of simplification (block, diagonal matrix) do not properly match the dynamic behaviour of the supporting structure.

\section{Conclusion}

This study has been performed to improve the accuracy in describe the dynamical behaviour of a whole rotordynamic assembly (including rotating and non rotating part). The paper especially focuses on the interaction between different bearing on the same shaft line caused by elastic supporting system. The study, analyses different modal reduction techniques and in particular the Component Mode Synthesis technique wich assures low time of calculation without compromising a good description of the model. It is also possible to note that the 
increasing level of approximation in the baseplates modelling may leads to an erroneous understanding of the interaction between the rotor and its support. The minimum level of accuracy in correctly describing the support structures necessary requires the coupling between bearings: the treediagonal model, that comprises cross-talking terms, is the minimal allowable description of the support structure that still guarantes the right behaviour of the FEM model. Future

development would be a comparison with other experimental data and the study of more complex rotor train.

\section{References}

1. U. Ehehalt, B. Luneburg, C. Daniel, J. Strackeljan e E. Woschke, Methods to Incorporate Foundation Elasticities in Rotordynamic Calculation, SIRM 2009 8th International Conference on Vibrations in Rotating Machines, 2009.

2. http://www.ansys.com

3. G. R. Cowper, The shear coefficient in timoshenko's beam theory,Journal of Applied Mechanics 33, 335-340 (1966) (6 pages); doi:10.1115/1.3625046

4. S K Park and X-L Gao, Bernoulli Euler beam model based on a modified couple stress theory, Journal of Micromechanics and Microengineering, 162355. doi:10.1088/0960-1317/16/11/015 (2006)

5. M. C. C. Bampton and R. R. Craig Jr, Coupling of substructures for Dynamic Analysis, AIAA Journal, vol. 6, no. 7, 1968.

6. P. Koutsovasilis and M. Beitelschmidt, Comparison of model reduction techniques for large mechanical systems, Multibody Syst Dyn (2008) 20: 111128 DOI $10.1007 / \mathrm{s} 11044-008-9116-4$ 